\title{
Theoretical optimization of quantum wire array lasers for low threshold current density and high modulation frequency
}

\author{
Igor Vurgaftman and Jasprit Singh \\ Department of Electrical Engineering and Computer Science, The University of Michigan, \\ Ann Arbor, Michigan 48109-2122
}

(Received 23 April 1993; accepted for publication 30 July 1993)

\begin{abstract}
We show that as one decreases the cross-sectional area of quantum wire lasers, the threshold current decreases, but the carrier relaxation time increases. Since the electron relaxation time sets the upper limit on the modulation frequency, there is a tradeoff between speed and efficiency in quantum wire lasers. We derive the optimal wire cross-sectional area for a one-dimensional array of quantum wire lasers based on a balance between an acceptably high maximum modulation frequency and a desirably low threshold current density. We find that for a relaxation time of $60 \mathrm{ps}$, the quantum wire of $150 \times 150 \AA$ cross section has the lowest threshold current density of $560 \mathrm{~A} / \mathrm{cm}^{2}$. If high-speed operation is not needed, the optimal choice for the quantum wire cross-sectional area is $100 \times 50 \AA$ with the threshold current density of 420 $\mathrm{A} / \mathrm{cm}^{2}$. For optimized quantum wells with the same cavity losses, the threshold current density is $\approx 620 \mathrm{~A} / \mathrm{cm}^{2}$. We also present the results for the threshold current density and the relaxation time that allow one to find the optimal quantum wire structure weighing the speed and efficiency considerations in accordance with their relative importance.
\end{abstract}

The density of states in quasi-one-dimensional (1D) electronic structures develops a strong peak near the band edge with the promise of yielding high gains at very low carrier injections. ${ }^{1-4}$ It has been demonstrated theoretically as well as experimentally that quantum wire lasers require lower threshold currents than their quantum well counterparts. ${ }^{5,6}$ On the other hand, the carrier relaxation processes in quantum wire structures appear to be slower than in quantum wells due to the reduction in momentum space to a single dimension and the resulting suppression of the major energy loss mechanism, polar optical phonon emission. ${ }^{7-11}$ Both effects become more prominent as the quantum wire cross section is decreased because of the reduction in the number of subband levels in the quasi-onedimensional system. Depending on the relative importance of the threshold current and modulation frequency considerations, the wire cross section can be optimized for a particular design of the quantum wire laser. In this letter, we optimize the quantum wire cross section for array lasers based on the theoretical calculations of the relaxation time and threshold current in quantum wires.

We consider a wire of GaAs of the lateral dimensions of the order of the deBroglie wavelength for electrons, bounded in the directions of confinement by the larger band gap barrier of $\mathrm{Al}_{0.3} \mathrm{Ga}_{0.7} \mathrm{As}$. A finite length of the wire ( $2000 \AA$ ) along the wire axis oriented in the [001] crystallographic direction is assumed in order to simplify the relaxation time calculation, however, the presented results are independent of its numerical value as long as it is much greater than the electron wavelength. From the point of view of the technological process, the fabrication of quantum wires oriented along the [011] direction is often easiest. The separation between the subbands in such a wire is greater than in the [001] wire, resulting in a lower density of states and more nearly parabolic valence band structure. Therefore, the [011] wire requires a lower threshold current, and the calculations for the [001] wire may be con- sidered as the lower bound on the performance of the [011] wires. On the other hand, the lower density of states may increase the relaxation times slightly for the [011] wires as compared with the [001] wires; however, since the error in defining the lateral dimension of the wire can be as great as $20 \AA$, the orientation dependence of the relaxation time is not expected to be a significant factor.

We assume the electron states have the central symmetry of $s$-type states and the hole states have that of $p$-type states and solve the band structure problem for the conduction and valence band in the $\mathbf{k} \cdot \mathbf{p}$ approximation. ${ }^{12,13}$ While the electron density of states in the well adheres to the expected one-dimensional negative square root functional behavior, the density of states in the valence band is quite complicated due to the mixing and coupling between heavy holes and light holes (Fig. 1). The nonparabolicities in the valence band make quantum wires

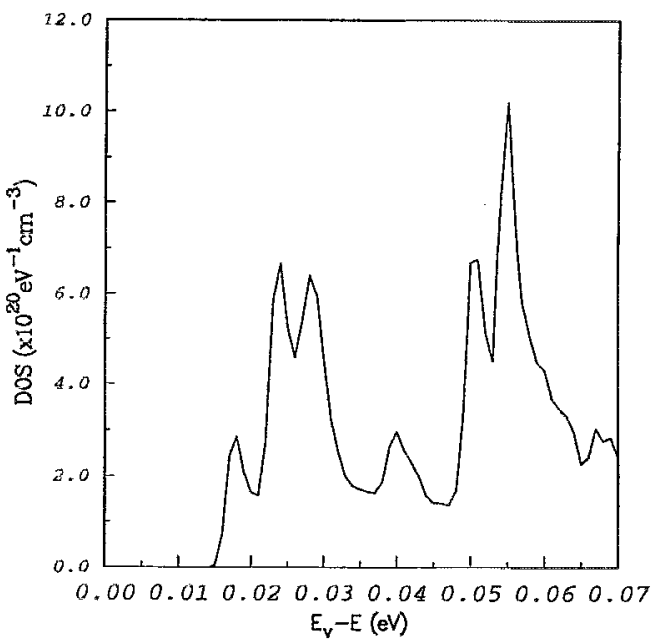

FIG. 1. The valence band density of states for the $100 \times 100 \AA$ quantum wire. 
a priori less attractive for laser applications, but an exact numerical analysis establishes the quantitative superiority of employing quasi-one-dimensional laser structures over the quantum wells. We calculate the material gain in quantum wires using the Fermi Golden Rule. It is given in Gaussian units by ${ }^{14,15}$

$$
\begin{aligned}
g(\hbar \omega)= & \frac{4 \pi^{2} e^{2} \hbar}{n_{0} c m_{0}^{2} \hbar \omega} \frac{1}{W L} \frac{2}{2 \pi} \int d k_{z} \sum_{n, m}\left|\hat{\varepsilon} \cdot \mathbf{P}_{n m}\left(k_{z}\right)\right|^{2} \\
& \times \delta\left[E_{n}^{e}\left(k_{z}\right)-E_{m}^{h}\left(k_{z}\right)-\hbar \omega\right] \\
& \times\left[f^{e}\left(E_{n}^{e}\right)-f^{h}\left(E_{m}^{h}\right)\right],
\end{aligned}
$$

where $\hat{\varepsilon}$ is the polarization of light, $\mathbf{P}_{n, m}$ is the momentum matrix element between the electron and hole states, $W$ is the width of the wire in the direction of current flow, $L$ is the length of the wire in the direction perpendicular to that of the current flow and to the wire axis, and $f^{e}\left(E_{n}^{e}\right)$ and $f^{h}\left(E_{m}^{h}\right)$ are the electron and hole quasi-Fermi functions, respectively. The calculation proceeds by adjusting the quasi-Fermi levels until the electron density equals the hole density in the active region. The delta function is represented by a Lorentzian broadening function with a $3-\mathrm{meV}$ linewidth. Light experiences optical gain only in the well region; therefore, we characterize the laser optical gain by multiplying the material gain by an optical confinement factor $\Gamma=\gamma W$, where $\gamma$ is the optical confinement per unit width and $W$ is the width of the quantum wire in the direction of current flow. The array is assumed to have a fixed length, the calculations are independent of its numerical value. The implication is that a $1 D$ array of a certain length will contain twice as many $100 \times 100 \AA$ as $200 \times 200$ $\AA$ wires. Our calculations are normalized with respect to this characteristic. We assume a typical value for $\gamma$ of 2.5 $\times 10^{-4} \AA^{-1}$ in GaAs-based lasers. The results are presented for a cavity loss of $48 \mathrm{~cm}^{-1}$. We normalize the model gain for quantum wires in an array of fixed length in the direction perpendicular to that of the flow of current. The peak model gain as a function of the $1 \mathrm{D}$ carrier concentration with the loss line superimposed on it is shown in Fig. 2. We obtain the threshold carrier densities of $\approx 2$ $\times 10^{6} \mathrm{~cm}^{-1}$ for the $100 \times 100 \AA$ wire, $6 \times 10^{6} \mathrm{~cm}^{-1}$ for the $150 \times 150 \AA$ wire, and $12 \times 10^{6} \mathrm{~cm}^{-1}$ for the $200 \times 200 \AA$ wire.

In order to determine the radiative current density, we must evaluate the spontaneous emission rate per unit area. This is done by computing the dipole transition rate for the quantum confined carrier states (Ref. 16) given in Gaussian units by

$$
\begin{aligned}
R_{\mathrm{sp}}= & \int d(\hbar \omega) \frac{4 e^{2} n_{0} \hbar \omega}{3 m_{0}^{2} c^{3} \hbar^{2}} \frac{1}{L} \frac{2}{2 \pi} \int d k_{z} \sum_{n, m}\left|\mathbf{P}_{n m}\left(k_{z}\right)\right|^{2} \\
& \times \delta\left[E_{n}^{e}\left(k_{z}\right)-E_{m}^{h}\left(k_{z}\right)-\hbar \omega\right]\left[f^{e}\left(E_{n}^{e}\right)\right] \\
& \times\left[1-f^{h}\left(E_{m}^{h}\right)\right] .
\end{aligned}
$$

The recombination rate normalized for an array of fixed length is shown in Fig. 3 for several quantum wire square cross sections. Now we evaluate the threshold current density by assuming that at threshold the carrier recombina-

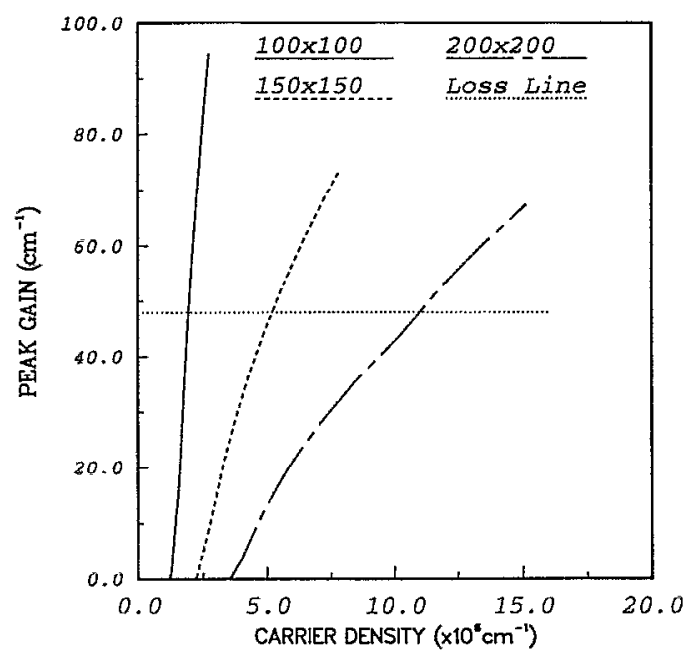

FIG. 2. Peak modal gain vs $1 D$ carrier concentration for several quantum wires. The intersection of the vertical loss line with the gain curves corresponds to the threshold condition.

tion is given by spontaneous emission. Then the total current density is $J_{\mathrm{th}}=e R_{\mathrm{sp}}\left(n_{\mathrm{th}}\right)$. This yields the following estimates for the threshold current density: $510 \mathrm{~A} / \mathrm{cm}^{2}$ for the $100 \times 100 \AA$ wire, $560 \mathrm{~A} / \mathrm{cm}^{2}$ for the $150 \times 150 \AA$ wire, and $740 \mathrm{~A} / \mathrm{cm}^{2}$ for the $200 \times 200 \AA$ wire. Reduction of the cross-sectional area decreases the required threshold current until the wire becomes so narrow that the penetration of the electronic states into the barrier reduces the electron-hole overlap. Since the lateral dimension of the wire is usually defined lithographically, it is expected to be difficult to reduce it below $100 \AA$. In view of these limitations, the optimum wire cross section that is potentially technologically realizable is $100 \times 50 \AA$ based on threshold current considerations alone. Such a wire requires a threshold current density of $\approx 420 \mathrm{~A} / \mathrm{cm}^{2}$. An optimized $\mathrm{GaAs}$ quantum well with a width of $50 \AA$ yields a value of $J_{\text {th }}$ $=620 \mathrm{~A} / \mathrm{cm}^{2}$ for identical cavity properties.

In order to optimize the wire cross section for high modulation frequency, we calculate the carrier relaxation time in quantum wires which sets the upper limit on the modulation frequency of the quantum wire laser. We per-

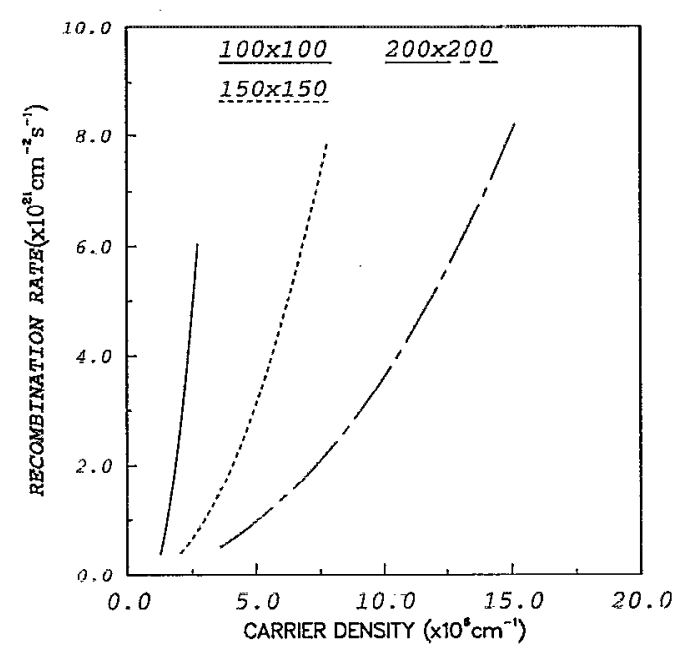

FIG. 3. Spontaneous recombination rate for several quantum wires. 


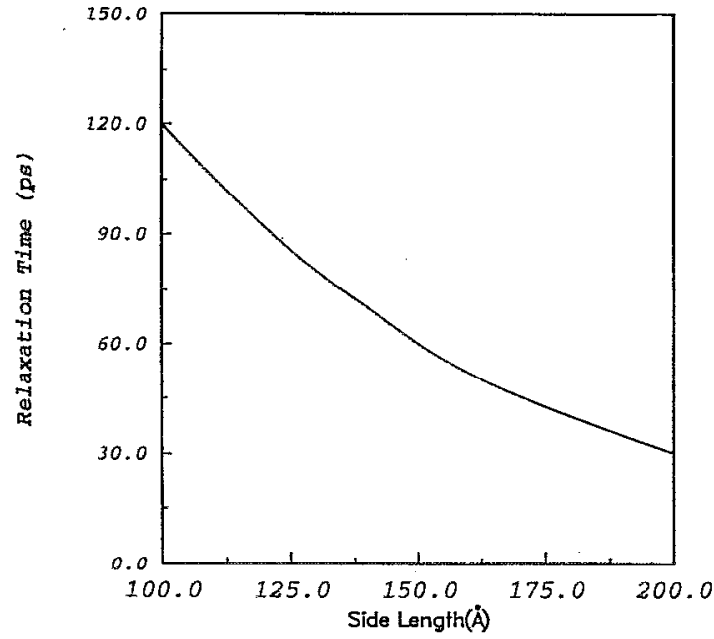

FIG. 4. Electron relaxation time as a function of the wire width for quantum wires of square cross section at room temperature and a carrier concentration of the order of $10^{18} \mathrm{~cm}^{-3}$.

form an ensemble Monte Carlo simulation of the time evolution of the electron distribution function. Since the hole confinement energy is much smaller than that for electrons, we consider electron relaxation alone. We inject carriers in a thermal distribution at the edge of the potential well formed by the barrier region and extract the relaxation time from the rate of energy loss by the electron distribution to the lattice at a constant temperature of $300 \mathrm{~K}$.

Since intrasubband electron-electron (EE) scattering in a quasi-1D system is prohibited by simultaneous conservation of energy and momentum, for wires of crosssectional areas less than $200 \times 200 \AA$ in which the subband spacing is greater than $k_{B} T$, EE scattering has little observable effect on the shape of the distribution function. The main scattering mechanism is polar optical phonon (POP) scattering calculated in the Born approximation from the Fermi Golden Rule. ${ }^{17}$ An emission of a POP is nearly four times as likely at $300 \mathrm{~K}$ as absorption; therefore, hot electrons lose their excess energy to the lattice relaxing to a quasi-Fermi distribution. Acoustic phonon scattering has mainly randomizing influence due to the high occupation number. Decreasing the temperature, reduces the phonon occupation, increases the fraction of emission events, and reduces the relaxation time making the energy loss mechanism more efficient. At high carrier concentrations, necessary to sustain population inversion in a semiconductor laser, the relaxation time is only a weak function of the carricr concentration. At carrier densities several orders of magnitude smaller, screening of the electron-phonon interaction is reduced drastically, and the relaxation processes are substantially faster. Details of the Monte Carlo calculation of the electron relaxation time in quantum wires have been presented elsewhere; ${ }^{10,11}$ we summarize the results by giving the equilibrium time as a function of cross section at $300 \mathrm{~K}$ in Fig. 4 .

The relaxation time decreases as the cross-sectional area of the quantum wire increases. For the $100 \times 100 \AA$ wire, the relaxation time is calculated to be $120 \mathrm{ps}$, for the $150 \times 150 \AA$ wire, it is reduced to $60 \mathrm{ps,} \mathrm{and} \mathrm{for} 200 \times 200$
$\AA$ to 30 ps. The relaxation time tends to the limit of a few picoseconds for bulk GaAs as the wire cross section is increased further. Based on the modulation scheme (direct or external) and the desired speed of the laser, these results provide quantitative information for optimization of the quantum wire laser design. For example, if a modulation frequency of $15 \mathrm{GHz}$ is desired with the lowest possible threshold current, a $150 \times 150 \AA$ quantum wire is the best choice. A $100 \times 100 \AA$ wire array will have a lower threshold current, but the modulation frequency will decrease by approximately $50 \%$. The results for a square cross section can be easily extrapolated to a more general rectangular cross section since the relaxation time depends on the cross-sectional area of the wire rather than on the individual lateral dimensions.

In conclusion, we have considered the issue of optimizing the quantum wire array lasers based on the threshold current density and modulation frequency. The threshold current density decreases while the maximum modulation frequency increases as the wire cross-sectional area is reduced. The relative prominence of speed and efficiency considerations dictates the correct choice of the wire cross section for any particular design. It appears that a quantum wire of the $150 \times 150 \AA$ cross section has the lowest threshold current for a modulation frequency of $15 \mathrm{GHz}$. The quantum wires have been demonstrated to have lower threshold current densities for the wire cross section less than $150 \times 150 \AA$ than the lowest threshold current densities achievable in quantum wells. The incorporation of strain in the wire region is expected to result in a further reduction of the threshold current, a behavior analogous to that observed in and calculated for the quantum well systems.

This work was carried out under a grant from the U.S. Army URI program DAAL 03-92-G-0109.

${ }^{1}$ Y. Arakawa and H. Sakaki, Appl. Phys. Lett. 40, 939 (1982).

${ }^{2}$ Y. Arakawa, K. Vahala, and A. Yariv, Appl. Phys. Lett. 45; 950 (1984).

${ }^{3}$ P. M. Petroff, A C. Gossard, R. A. Logan, and W. Wiegman, Appl. Phys. Lett. 41, 635 (1982).

${ }^{4}$ M. Tsuchiya, M. J. Gaines, R. H. Yan, R. J. Simes, P. O. Holtz, L. A. Colden, and P. M. Petroff, Phys. Rev. Lett. 62, 466 (1989).

${ }^{5}$ S. Tiwari, G. D. Pettit, K. R. Milkove, R. J. Davis, J. M. Woodall, and F. Legoues, IEDM Tech. Dig. 859 (1992).

${ }^{6}$ I. Vurgaftman, J. M. Hinckley, and J. Singh, IEEE J. Quantum Electron. (to be published).

${ }^{7}$ J. P. Leburton, Phys. Rev. B 45, 11022 (1992).

${ }^{8}$ V. B. Campos and S. Das Sarma, Phys. Rev. B 45, 3898 (1992).

${ }^{9}$ V. B. Campos, S. Das Sarma, and M. A. Struscio, Phys. Rev. B 46, 3849 (1992).

${ }^{10} \mathrm{I}$. Vurgaftman and J. Singh, Appl. Phys. Lett. 62, 2251 (1993).

${ }^{11} \mathrm{I}$. Vurgaftman and J. Singh (unpublished).

12 J. M. Luttinger and W. Kohn, Phys. Rev. 97, 869 (1955).

${ }^{13}$ S. Hong, M. Jaffe, and J. Singh, IEEE J. Quantum Electron. QE-23, 2181 (1987).

${ }^{14}$ Y. Lam, J. P. Loehr, and J. Singh, IEEE J. Quantum Electron. QE-28, 1248 (1992).

${ }^{15} \mathrm{~J}$. Singh, Physics of Semiconductors and Their Heterostructures (McGraw-Hill, New York, 1993).

${ }^{16}$ J. J. Sakurai, Advanced Quantum Mechanics (Addison-Wesley, New York, 1967).

${ }^{17}$ B. K. Ridley, Quantum Processes in Semiconductors (Clarendon, Oxford, 1982). 